\title{
COGNITIVE AND EMOTIONAL EFFECTS OF BILINGUALISM CHILDHOOD AND ADULTHOOD
}

\author{
Umi Farisiyah \\ Madrasah Aliyah Al Hikmah 2, Brebes, Central Java, Indonesia, farisiyahumi@ yahoo.com
}

\begin{abstract}
Mastering more than one languages gives some advantages because of the correlation of the language and the brain. If we use more than one languages mean our brain has to work more that it works in one language. It means our brain has to be more active that can activate all the neurons of the brain and automatically make the brain much better in its functions. And of course, using more than one languages change how we assume and interpret everything in our surrounding. Using evidences from reaction time, eye-tracking, functional magnetic resonance imaging, and event-related potential research, it shows how language experience transforms cognitive control and emotion, two features that they are central to human communication. We suggest that managing multiple languages not only impacts cognitive control and emotion independently, but also the way in which they interact with each other. Within cognitive control, we present research suggesting that bilinguals demonstrate benefits compared to monolinguals and discuss the potential sources of these benefits, including parallel language activation and language switching. We also explore the potential links between linguistic and non-linguistic cognitive processes. Within emotion, we discuss whether bilinguals process emotions similarly across their first and second languages and consider how linguistic context and one's cultural affiliation may impact memory (e.g., storage and retrieval) of emotional events. The evidence discussed in this chapter highlights the transformative effect that bilingual experience has on how human beings understand and interpret the world.
\end{abstract}

Keywords: cognitive control, inhibitory control, emotion, bilingualism, biculturalism

Language is ability that human being has as the distinction from other creatures. This device helps human to be capable of shaping thoughts and perceptions toward everything happened, heard, seen, experienced, as well as got in this life. Language is really helpful for us. At our social life, it is a very important device for us to have a communication with others, to state what are in our mind, and to share what we feel or what think. Indeed, at a cognitive level, language also has a huge impact in understanding, comprehending all events, experiences and activities in our environment, and bridge the meaning from every single of them within our minds. For monolinguals who only use one language will be limited by their own language when they are in different language communities, or on the other hand, their understanding, thought and ability to communicate are restricted compared with bilinguals who have broader those abilities. So, bilinguals have different abilities, in positive sides, than monolinguals due to the influence of mastering more than one language on their cognitive system. The correlated neuroimaging (fMRI scans) of bilinguals showed a greater activity in the prefrontal cortex networks, which direct the highest levels of thinking and awareness, directing these and other executive functions. (Bialystok, 2009; Kaushanskaya \& Marian, 2007). As we have already known that language is stuck together with our brain. Two important areas on our brain dealing with language are Broca's area and Wernicke's area. If we see the visualization of bilinguals' brain on the two areas are wider because bilinguals' two language areas are always active. This is so much different from monolinguals' brain visualization.

Two hemispheres are involved when we are using a language, they are right and left hemisphere. Left hemisphere is a dominant or superior hemisphere on language; it has important roles on language 
and has verbal memory function, whereas the right hemisphere is for emotional function. Using language without involving the right hemisphere will not have intonation, prosody, melody and emotion. It explains that language use two hemispheres of the brain. Using language has inter-correlation between two cognitive system; cognitive control and emotion. Take this for the example, when the bilinguals are having a chat with our friends in a crowded place or in a place that uses a different language from them, they will have a control to manage the responses should or should not be responses. This control ability is called inhibitory control dealing with cognitive control. Additionally, when the bilinguals asked to describe about their memorable experiences, the language they produce will be tightly bound to the emotion associated with the experience. These two examples give illustration on how cognitive control and emotion are connected and how these aspects of cognition are filtered through and by language. By mastering two or three (more than one) languages may change how we attend to the environment due to its function of the language dealing with cognitive control, memory and emotion. What we think and feel about everything we experience may be formed by our language.

Neurobilingual research suggests a link between cognitive control and emotional processes in the brain. The regions involved in cognitive control (e.g. attention, self- regulation), prefrontal cortex (PFC) and anterior cingulate cortex (ACC) and emotion become activated when situations demand emotion regulation or control of emotional thoughts, such as in response to an aversive event (e.g. pain). Interestingly, bilinguals simultaneously activate areas associated with cognitive control and emotion on language switching tasks, even when processing emotionally-neutral words. (Hernandez, 2009).

The evidence suggests that the bilingual mind may be organized differently than single-language minds (Kroll \& Bialystok, 2013, pp. 497-498). Experiences with multiple languages may also result in an enriched cultural identity that incorporates elements from both cultures, which may transform bilinguals' mental conceptual structure and the way they process their environment. These rich language and cultural experiences further impact cognition, resulting in enhanced cognitive control abilities, and shaping how emotional events are processed and retrieved from memory.

In the current chapter will be presented evidence that highlights how and why bilingual experience impacts the human mind. Though the effects of bilingual experience on cognition are wideranging, only two aspects of cognition: cognition control and emotion, as well as how these aspects interact. The two impacts from the bilingual experience will be focused on the bilingual experiences in childhood, adolescent and adulthood, because in every level of development, the effects of bilinguals will not be the same due to the function of the language its self and also the development tasks of the three levels mentioned that are so much different.

\section{Bilingualism and Cognition}

Cognition has meant different things to different people at different times. In order to explore the field of cognition, we need some kind of map of the territory. One map is used to examine the ways that the word cognition has been used. This is the approach that was successfully taken by Natsoulas (1978) when he explored the concept of consciousness. Notsoulas pointed out that scientific psychology develops out of the concerns of everyday life. Notsoulas used The Oxford English Dictionary as a guide to the various meanings of consciousness. The Oxford English Dictionary lists several meanings of cognition, including the action or faculty of knowing. This definition emphasizes two things. One point worth emphasizing is that cognition is the action of knowing. The study of cognition is the study of processes: the ways in which we became acquainted with things. The second point of emphasis is that cognition can be seen as a faculty. As we shall see, it has been common to divide the mind up into faculties that represent the different mental activities of which we are capable. (Benjafield, 1992: 2). 


\section{Bilingualism and Cognitive Control}

Cognitive control is the way to manage distraction or conflicting information in our lives and this is related with the skills that help people to navigate their environment. There are three aspects including in cognitive control, they are conflict monitoring which is the ability to keep track of conflict or potential conflict in the environment, competition resolution or the ability to manage and resolve distractions while trying to focus on relevant information and interference suppression and response inhibition which involve ignoring distracting information or viable alternative responses when responding to a direction or question. Cognitive control is not limited to a single mental process, like language or vision, but extends into every aspect of perception and cognition.

\section{Effects of Bilingualism on Language Activation and Interaction}

When doing a conversation whether speaking or listening, bilinguals activate both of their languages simultaneously. This parallel activation happens across different linguistic contexts for bilinguals (Kroll \& Bialystok, 2013) including in language perception and production. When both areas are active, bilinguals have to manage the activation of an irrelevant language while trying to understand and produce speech in the target language. This causes the increase of Bilinguals' overall cognitive load. To do this management, bilinguals rely on inhibitory control. Inhibitory control is a device to choose what language that the speaker should produce, in this case, sometimes, switching languages often happens. By using inhibitory control more often makes this device more sensitive and make the brain does too. When using one language, another area of language is also active. It makes more neurons on two parts of brain work. That thing is so much in line with the research that states the more our brain works, the better its function is. Thus being natural a "mental-Juggler" of two languages (Kroll, 2009) increases cognitive control efficiency and transform the cognitive system, allowing it to accommodate and manage multiple languages.

Evidence from language switching studies may also offer an explanation as to why inhibitory control is more efficient in bilinguals. Bilinguals who are less proficient in their language target must inhibit their native language (L1) more strongly in order to avoid interference while using their target language (L2). It draws that the inhibitory control in bilinguals are more selective in selecting the language.

During language switching task, both languages in a bilingual are activated in parallel and compete for selection. The positive effects of bilingualism on language activation and interaction to children are making their brain improving progress is so much optimal due to the activeness of the brain. Besides that, Bilingual children outperform monolingual children on tasks that require inhibiting interfering task-incongruent information (Barac \& Bialystok, 2011; Martin-Rhee \& Bialystok, 2008) and also allo-cate their visuospatial working memory resources more efficiently than monolinguals (Bialystok, 2010; Morales, Calvo, \& Bialystok, 2013).

\section{Effects of Bilingualism on the Interaction between Linguistic and Non-Linguistic Cognitive Control}

Parallel activation and language switching in bilinguals require the use of linguistic cognitive control. More specifically, the link between language and cognition in bilinguals has led to a growing line 
of research exploring the effects of parallel language activation and linguistic and non-linguistic inhibitory control.

Although bilinguals and monolinguals showed similar within-language competition, bilinguals demonstrated less competitor inhibition on the subsequent priming probe trials. Suggesting that bilinguals overcome linguistic interference more quickly and efficiently than monolinguals. Freeman, Shook, and Marian found that in bilinguals but not in monolinguals better performance on this task was related to better resolution of linguistic competition. These findings demonstrate a relationship between non linguistic cognitive control and management of linguistic competition in bilinguals as well as the impact of language experience on cognitive control.

The further explore relationship between language activation and cognitive control, in a subsequent study, Blumenfeld and Marian (2013) measured between-language competition and cognitive control abilities in bilinguals. The cognitive benefits may arise from the need to manage switching between two languages that are activated in parallel. Importantly, the studies discussed in this section demonstrate that bilinguals can be considered experts at managing linguistic competition.

In one study, researchers taught babies growing up in monolingual or bilingual homes that when they heard a tinkling sound, a puppet appeared on one side of a screen. In order to get reward, the infants had to adjust the rule they had learned; only bilingual babies were able to successfully learn the new rule. This suggests that even for very young children, navigating a multilingual environment imparts advantages that transfer beyond language. The possible explanation on this study is the effect of bilingualism between the interactive of the non-linguistic cognitive control. Even only use a sound, the bilingual babies can understand and learn the new rules.

\section{Effects of Bilingualism on Emotion Processing}

Bilingualism also impact another higher order aspect of cognitive system that is Emotion. It has been suggested that across the world's languages, some concepts, words and emotion may not transfer (Pavlenko, 2008) and between bilingual, we may see a "lost in translation" effect when attempting to express or process an emotion for which there is no label in one of his/ her language. A possible explanation for the difference in the first language and the second language emotional word processing is that adult of second language learners process second language emotional words at a more semantic rather than affective level (Pavlenko,2012). It means that emotional words are easy to be remembered by the learners. This also happens to women who have a unique way to reverse their memory that is by always connecting it with emotion.

Ferre, Garcia, Fraga, Sanchez-Casas, and Molero (2010) tested a memory of emotional words across three bilinguals groups (early Catalan-Spanish bilinguals who were Catalan dominant, early Spanish-Catalan Bilinguals who were Spanish dominant and late Spanish-English bilinguals). All the three bilinguals groups recalled emotional words equally well in both languages, and factors such as age acquisition, dominance, and similarity between language pairs (Spanish-English versus Spanish-Catalan) did not affect bilinguals recall ability. Ferre et al. also suggested that, for proficient bilinguals, emotional words in the memory task had the same intensity or impact across the first language and the second language, supporting the notion that as the second language proficiency increased, so does the processing equivalence of emotional words across language. (Freeman, 2016). 
The emotional word processing across languages also comes from an investigation using the emotional Stroop task with bilinguals (Sutton Altarriba, Gianico, \& Basnight-Brown, 2007). Emotional words typically result in slower response latencies than neutral words. The emotional Stroop task requires participants to name the colour that a word is printed in (e.g. red, blue, green, brown or black ink). Unlike the traditional Strop task, there is no incongruent condition (e.g. left arrow pointing to the right, or naming BLUE when it is presented in red ink) and thus no incongruence effect. Instead, participants view both emotional and neutral words (e.g. they may see the word EXAM written in green ink, and would respond by saying green); emotional words typically result in slower response latencies than neutral words. Sutton et al. (2007) found that balanced Spanish-English bilingual reaction times to emotional words were slower that reaction times to neutral words regardless of the language presentation. In other words, the bilinguals in Sutton et al. (2007) processed emotional words equally across both the first / native language and the second/ target language. These findings are consistent with the Revised Hierarchical Model (Kroll \& Stewart, 1994), which would predict that for balanced bilinguals, the native language and the target language translation are strongly connected to a shared conceptual representation, and balanced bilingual can thus access the affective meaning of each word equally.

In contrast, there is also evidence suggesting that emotional words may be processed similarly across the first language and the target language regardless of proficiency. All there bilinguals groups recalled emotional words equally well in both languages, and factors such as age of acquisition, dominance and similarity between language pairs did not affect bilinguals' recall ability.

One way to reconcile conflicting findings of emotion processing across languages is to consider that although emotion processing in the native language may be more automatic than in the target language. The quality of the emotional experiences may be similar across both languages. Language experiences, specifically proficiency also appears to play a role in the degree to which emotional words are processed similarly across the fire and second languages, with balanced bilinguals processing the native language and the target language emotional words more.

Bilingual people often perform better on tasks that require conflict management, as it is stated in the classic Stroop task. And they are also better at switching between two tasks. From the two results written, it can be concluded that these two abilities also can be applied on managing emotion. Bilinguals often face conflicts on selecting language that should or should not be produced. It means, in relating with emotion, bilinguals (both child and adult bilinguals) are able to express their appropriate emotion on problems they are facing. On the other hand, they are selective in showing their emotion toward everything they face. They are also better in switching the two languages they mastered means that bilinguals are easy to adapt on the environment they stand in.

\section{Effects of Bilingualism on Emotional Memory}

Previous work on memory retrieval in bilinguals demonstrates that a memory is better expressed in the language in which it was encoded at the time the event took place (Marian \& Fausey, 2006; Marian \& Kaushanskaya, 2007; Marian \& Neisser, 2000). Not only can the language of retrieval impact how well the event is expressed, but the language of retrieval can also affect the emotional content of the memory. In Marian and Kaushanskaya (2008), Russian- English bilingual participants were asked to describe (via narrative samples) their memories of immigration to the U.S. in either Russian or English to a Russian-English bilingual experimenter. The results demonstrated that the Russian-English bilinguals produced more emotional words in second language narratives. In addition to differences in emotionality across languages, the results of Marian and Kaushanskaya (2008) were impacted by age of immigration. When speaking Russian, immigrants who came to the U.S. from Russia early in life 
produced longer narratives, more positive emotion words, and used more emotional words overall than immigrants who arrived later. That is, bilinguals who immigrated earlier reconstructed their memory of the event based off of current experiences with the target language culture, which tended to be more positive. For the children who are bilinguals, they tend to choose the language that they want to use that time is the language that is easier or having emotionally closed to them, as like simple or unique pronunciation.

\section{Effects of Biculturalism on Emotion}

In addition to the interplay between language, memory, and emotion, language and culture are closely intertwined (Freeman, 2016). When exploring how bilingual experience impacts emotion, it is necessary to consider cultural influences, as culture imposes norms on how we interpret emotional events. Marian and Kaushanskaya (2004) explored the variability of the type of emotional words used to describe events across languages (and cultures) in Russian-English bilingual immigrants who came to the U.S. from the former Soviet Union. Participants were asked to describe two separate events (e.g., birthday, snowfall), one in each language. Pronoun usage was used to measure cultural influence: collectivism (i.e., emphasis on the group: former Soviet Union) was measured by the increased use of group pronouns, like "we," and individualism (i.e., emphasis on the individual: U.S.) was measured by the increased use of individual pronouns, like "I."

For children who are bilinguals also can show this emotional effect of bilingualism. For the example, when the children' first language is slower and longer intonation and the second language intonation is straight and rough, they can differentiate in using the intonation when they want to speak to what kind of language the person use in their communication. This difference happens because of the influence of the culture of the language.

The results from Marian and Kaushanskaya (2004) suggest that the linguistic context and the culture with which one affiliates influence the organization of emotions and retrieval of memories within the cognitive system. The possible explanation for this is because of cognitive control and emotion work together. There are in line and connected. So, bilinguals do not only choose the appropriate language they are supposed to use but also appropriate emotion they have to show.

Evidence from processing, memory, and cultural effects of bilingualism on emotion demonstrates that the accessibility and processing of emotion is highly complex and influenced by language experience. Because language, emotion, and memory are tightly linked within the cognitive system, we also suggest that language usage drives bilinguals to remember events based on linguistic cues, and that memories themselves may store information or cues about the language, and by extension, the culture.

\section{EFFECTS OF BILINGUALISM ON THE INTERSECTION OF COGNITIVE CONTROL AND EMOTION}

While bilingual experience impacts cognitive control and emotion independently within the cognitive system, experience with multiple languages can also shape how cognitive control and emotion interact with each other. In addition to research from Blumenfeld and colleagues suggesting that bilinguals activate their languages in parallel $(2007,2013)$, the valence of an emotional word also affects a bilingual's ability to activate or access its first language translation equivalent when processing that emotional word in target language. Storbeck and Clore (2007) stated that a study about emotion and cognition must be integrated because those phenomena are already integrated. They added that some different emotions do not have their own area in the brain, but they emerge from a combination of effective and cognitive processes.

Further evidence highlighting the interactivity between cognitive control and emotion comes from neural network research on humans and non-human animal models. Common to cognitive control and emotion is the anterior cingulate cortex (ACC), which may be involved in error detection and correction, and is activated during tasks that involve cognitive control and tasks that involve emotion processing (Bush, Luu, \& Posner, 2000). 
It is clear that the neural network sub serving cognitive control and emotion is highly integrated in humans, but what remains unclear is how this network is impacted by experience with managing multiple languages. In a language switching task, bilinguals activated cognitive control regions and also activated regions central to emotional processing (Hernandez, 2009).

Some previous studies have shown the activation of cognitive control regions during emotional tasks and events, Hernandez (2009) demonstrates that bilinguals can activate brain areas associated with emotion during an emotionally-neutral linguistic/cognitive control task. The underlying cause for activation of overlapping areas involved in cognitive control and emotion in bilinguals remains unclear and is subject to future neuroimaging research. However, the evidence discussed in this section clearly suggests that experience with multiple languages affects the interplay between cognitive control and emotion.

\section{Conclusion}

Bilinguals are thought to be "mental jugglers" (Kroll, 2009), constantly having to navigate both of their languages, even under contexts in which only one language is required. The demands imposed by parallel activation and language switching appear to confer benefits to bilinguals in both linguistic and non-linguistic contexts. As reviewed in this chapter, bilinguals demonstrate advantages in cognitive control. Brain pathways and networks that are responsible for cognitive control and language control overlap in bilinguals, and the cognitive control network is enhanced and recruited more efficiently in bilinguals than in monolinguals during language processing.

Likewise, eye-tracking research demonstrates that linguistic and non-linguistic cognitive control are correlated in bilinguals (Blumenfeld \& Marian, 2013), suggesting a tight relationship between language and cognition.

Research on bilingualism has also shown a complex relationship between linguistic experience and emotional processing, where a bilingual's ability to access the emotional content of words or memories depends on the language being used at a given time. For instance, there is mixed evidence as to whether bilinguals process emotional content similarly across their languages. In unbalanced bilinguals, emotional word processing may be more automatic in the first language than in the second language, but how emotion is represented in the brain may be similar across both languages. In addition, evidence from bilinguals also demonstrates how emotion influences the encoding and retrieval of memories.

Marian and Kaushanskaya (2004) showed high interactivity between emotion and memory, and experience with multiple languages affects how bilinguals form, store, and retrieve memories. Our emotional perception of an event is influenced by the linguistic and cultural context in which the event takes place, as well as the language in which the emotional event is retrieved.

We conclude that language, cognitive control, and emotion are intertwined. Indeed, cognitive control and emotion appeared to be both controlled by language. Our perception of emotional experiences relies on cognitive control to manage emotional reactions, and may use language to encode and express these reactions. B ilingual experience is unique in that knowing multiple languages can have advantageous effects on the cognitive system, altering the interactivity between cognitive control and emotion, as well as affecting each of these components independently. Experience with multiple languages can change how we think about and interpret daily events in the environment, allowing for more than one channel through which we view the world. 


\section{References}

Benjafield. (1992). Cognition. New Jersey: Prentice Hall Inc.

Berns, Margie. (2010). Concise Encyclopedia of Applied Linguistics. London: Elsevier Ltd.

Bialystok, E., Craik, F. I. M., \& Luk, G. (2008). Cognitive control and lexical access in younger and older bilinguals. Journal of Experimental Psychology: Learning, Memory, and Cognition, 34(4), 859873.

Bialystok, E., Craik, F. I. M., Klein, R., \& Viswanathan, M. (2004). Bilingualism, aging, and cognitive control: Evidence from the Simon task. Psychology and Aging, 19, 290-303.

Bialystok, E., Martin, M. M., \& Viswanathan, M. (2005). Bilingualism across the lifespan: The rise and fall of inhibitory control. International Journal of Bilingualism, 9(1), 103-119.

Blumenfeld, H. K., \& Marian, V. (2007). Constraints on parallel activation in bilingual spoken language processing: Examining proficiency and lexical status using eye-tracking. Language and Cognitive Processes, 22(5), 633-660.

. (2013). Parallel language activation and cognitive control during spoken word recognition in bilinguals. Journal of Cognitive Psychology, 25(5), 547- 567.

Bush, G., Luu, P., \& Posner, M. I. (2000). Cognitive and emotional influences in anterior cingulate cortex. Trends in cognitive sciences, 4(6), 215-222.

Ferré, P., García, T., Fraga, I., Sánchez-Casas, R., \& Molero, M. (2010). Memory for emotional words in bilinguals: Do words have the same emotional intensity in the first and in the second language? Cognition and Emotion, 24(5), 760-785.

Freeman, Max R., Shook, A., and Marian, V. (2016) Cognitive and emotional effects of bilingualism in adulthood. Lifespan Perspectives on Bilingualism.

Hernandez, A. E. (2009). Language switching in the bilingual brain: What's next? Brain and Language, 109(2), 133-140.

Kroll, J. F. (2009). The consequences of bilingualism for the mind and the brain. Psychological Science in the Public Interest, 10(3), $\mathrm{i}-\mathrm{ii}$.

Kroll, J. F., \& Bialystok, E. (2013). Understanding the consequences of bilingualism for language processing and cognition. Journal of Cognitive Psychology, 25(5), 497-514.

Kroll, J. F., \& Stewart, E. (1994). Category interference in translation and picture naming. Evidence for asymmetric connections between bilingual memory representations. Journal of Memory and Language, 33(2), 149-174.

Ling, Jonathan \& Catling, J. (2012). Cognitive Psychology. London: Pearson Education Limited.

Marian, V., \& Kaushanskaya, M. (2004). Self-construal and emotion in bicultural bilinguals. Journal of Memory and Language, 51(2), 190-201.

(2007). Language context guides memory content. Psychonomic Bulletin \& Review, 14(5), 925-933.

Marian, V., \& Neisser, U. (2000). Language-dependent recall of autobiographical memories. Journal of Experimental Psychology General, 129(3), 361-368. 
Martin-Rhee, M. M., \& Bialystok, E. (2008). The development of two types of inhibitory control in monolingual and bilingual children. Bilingualism: Language and Cognition, 11(01), 81- 93.

Pavlenko, A. (2008). Emotion and emotion-laden words in the bilingual lexicon. Bilingualism: Language and Cognition, 11(2), 147-164.

. (2012). Affective processing in bilingual speakers: Disembodied cognition? International Journal of Psychology, 47(6), 405-428.

Schunk, D.H. (2012). Learning Theories. London: Pearson Education Limited.

Sutton, T. M., Altarriba, J., Gianico, J. L., \& Basnight-Brown, D. M. (2007). The automatic access of emotion: Emotional Stroop effects in Spanish-English bilingual speakers. Cognition and Emotion, 21(5), 1077-1090. 